\title{
Sistemas de Informação em Saúde no apoio à gestão da Atenção Primária à Saúde: revisão integrativa
}

\section{Health Information Systems to support Primary Health Care management: an integrative review}

\section{Sistemas de Información de Salud para apoyar la gestión de la Atención Primaria de Salud: revisión integradora}

José Erivelton de Souza Maciel Ferreira ${ }^{1, a}$

eriveltonsmf@live.com | http://orcid.org/o0o0-0003-2668-7587

Lídia Rocha de Oliveira ${ }^{1, a}$

lidiarocha@aluno.unilab.edu.br | http://orcid.org/oooo-0001-7716-1388

Wanderson Souza Marques ${ }^{1, a}$

wamqs09@gmail.com | http://orcid.org/0000-0002-9619-2363

Talita Silva de Lima $^{1, a}$

talita lima.18@hotmail.com | http://orcid.org/o0oo-0001-7420-184X

Eloise da Silva Barbosa ${ }^{1, a}$

eloise.barbosa@hotmail.com | http://orcid.org/0000-0003-3312-2875

Révia Ribeiro de Castro ${ }^{2, b}$

revia_ribeiro@hotmail.com | http://orcid.org/0000-0002-9260-4148

José Maria Ximenes Guimarães ${ }^{3, c}$

jm_ximenes@hotmail.com | http://orcid.org/0000-0002-5682-6106

${ }^{1}$ Universidade da Integração Internacional da Lusofonia Afro-Brasileira, Instituto de Ciências da Saúde. Redenção, CE, Brasil.

2 Universidade Estadual do Ceará, Programa de Pós-Graduação em Cuidados Clínicos em Enfermagem e Saúde. Fortaleza, CE, Brasil.

${ }^{3}$ Universidade Estadual do Ceará, Programa de Pós-Graduação em Saúde da Família. Fortaleza, CE, Brasil.

a Graduando em Enfermagem pela Universidade da Integração Internacional da Lusofonia Afro-Brasileira.

b Mestrado em Saúde e Sociedade pela Universidade do Estado do Rio Grande do Norte.

c Doutorado em Saúde Coletiva pela Universidade Estadual do Ceará.

\section{RESUMO}

O objetivo deste estudo foi analisar a utilização dos Sistemas de Informação em Saúde para a construção da sala de situação na Atenção Primária à Saúde. Trata-se de uma revisão integrativa realizada por meio de busca de artigos na íntegra, nas bases de dados BDENF, Lilacs, Medline, SciELO, PubMed e Cochrane. Foram analisados sete artigos, os quais foram apresentados em três categorias. Os sistemas de informação apoiam a sala de situação na coleta, no processamento e na divulgação de resultados, na análise e na comparação de indicadores, no planejamento, na gestão e na avaliação em saúde. Por fim, percebeu-se que os sistemas de informação contribuem para a construção da sala de situação subsidiando a atuação da gestão local na formação de indicadores de saúde e na implementação de ações.

Palavras-chave: Sistemas de Informação em Saúde; Diagnóstico da situação de saúde; Atenção Primária à Saúde; Gestão em saúde; Avaliação em saúde. 


\section{ABSTRACT}

The aim of this study was to analyze the use of Health Information Systems in the construction of the situation room in Primary Health Care. This is an integrative review performed by searching full articles in the BDENF, Lilacs, Medline, SciELO, PubMed and Cochrane databases. Seven articles were analyzed and presented in three categories. Information systems support the situation room in the collection, processing and dissemination of results, in the analysis and comparison of indicators, and in the health planning, management and evaluation. In the end, we realized that information systems contribute to the construction of the situation room, supporting local management actions in the establishment of health indicators and the implementation of actions.

Keywords: Health Information Systems; Diagnosis of health situation; Primary Health Care; Health management; Health avaluation.

\section{RESUMEN}

El objetivo de este estudio fue analizar el uso de los Sistemas de Información de Salud en la construcción de la sala de situación en Atención Primaria de Salud. Esta es una revisión integradora realizada mediante la búsqueda de artículos completos en las bases de datos BDENF, Lilacs, Medline, SciELO, PubMed y Cochrane. Siete artículos fueron analizados y presentados en tres categorías. Los sistemas de información apoyan la sala de situación en la recolección, lo procesamiento y la difusión de resultados, en la análisis y la comparación de indicadores, y en la planificación, gestión y evaluación de la salud. Finalmente, se dio cuenta de que los sistemas de información contribuyen a la construcción de la sala de situación, apoyando las acciones de gestión local en lo establecimiento de indicadores de salud y la implementación de acciones.

Palabras clave: Sistemas de Información en Salud; Diagnóstico de la situción de salud; Atención Primaria de Salud; Gestión en salud; Evaluación en salud.

\section{Contribuição dos autores:}

Concepção e desenho do estudo: José Erivelton de Souza Maciel Ferreira, Lídia Rocha de Oliveira, Wanderson Souza Marques, Talita Silva de Lima, Eloise da Silva Barbosa, Révia Ribeiro de Castro, José Maria Ximenes Guimarães. Aquisição, análise ou interpretação dos dados: José Erivelton de Souza Maciel Ferreira, Lídia Rocha de Oliveira, Wanderson Souza Marques, Talita Silva de Lima, Eloise da Silva Barbosa, Révia Ribeiro de Castro. Redação do manuscrito: José Erivelton de Souza Maciel Ferreira, Lídia Rocha de Oliveira. Revisão crítica do conteúdo intelectual: José Maria Ximenes Guimarães.

Declaração de conflito de interesses: não há.

Fontes de financiamento: não houve.

Considerações éticas: não há.

Agradecimentos/Contribuições adicionais: não há.

Histórico do artigo: submetido: 3 set. 2019 | aceito: 12 ago. 2020 | publicado: 17 dez. 2020.

Apresentação anterior: não houve.

Licença CC BY-NC atribuição não comercial. Com essa licença é permitido acessar, baixar (download), copiar, imprimir, compartilhar, reutilizar e distribuir os artigos, desde que para uso não comercial e com a citação da fonte, conferindo os devidos créditos de autoria e menção à Reciis. Nesses casos, nenhuma permissão é necessária por parte dos autores ou dos editores. 


\section{INTRODUÇÃO}

Os Sistemas de Informação em Saúde (SIS) se configuram como uma estrutura capaz de garantir a obtenção e a transformação de dados em informação. Assim, as informações são coletadas, processadas, armazenadas e partilhadas com o objetivo de auxiliar no processo decisório em saúde, uma vez que contribui na produção de conhecimentos sobre as condições de vida e saúde da população, sobre morbimortalidade e seus fatores de risco, entre outros. Assim sendo, a utilização dessas informações permite a otimização dos processos gerenciais e assistenciais, inclusive no cuidado direto ao paciente ${ }^{1}$, o que possibilita o direcionamento de ações e recursos no enfrentamento dos problemas sanitários ${ }^{2}$.

No Brasil, o Ministério da Saúde deu início à organização dos SIS em 1975-1976, quando instituiu o Sistema de Informação sobre Mortalidade (SIM), que somente na década de 1990 foi descentralizado, com maior aporte de investimentos na sua informatização. Nas últimas décadas, foram criados vários SIS, com vistas a favorecer a disponibilidade de informações de saúde em tempo oportuno, mediante a construção de indicadores que permitam a análise da situação de saúde nos âmbitos local, estadual e nacional. Entre os principais sistemas de informação, operados por esse órgão oficial brasileiro, destacam-se: o ambulatorial (SIA-SUS), o de internações hospitalares (SIH-SUS), o de notificação de doenças e agravos à saúde (Sinan), o de mortalidade (SIM), o de nascimento (Sinasc), o de atenção básica (Sisab) - todos esses consideram níveis populacionais. No que se refere aos serviços de saúde, tem-se o Cadastro Nacional de Estabelecimentos de Saúde (CNES), que é a principal fonte de dados sobre serviços públicos e privados³.

Destaca-se o fato de que esses sistemas de informação, de base nacional, passaram a ser de alimentação obrigatória, para estados e municípios, com regulamentação em portarias ministeriais, que vinculam seu cumprimento ao repasse de recursos financeiros aos municípios. Assim, a gestão é compartilhada, competindo ao Departamento de Informática do SUS (Datasus) manter o acervo de dados e difundi-los aos gestores e ao público em geral ${ }^{4}$.

Com efeito, o arcabouço normativo do Sistema Único de Saúde (SUS) estabelece que a organização dos serviços de saúde se deem com base no planejamento ascendente, desde o nível local ao nacional, pautado na análise da situação de saúde da população, o que torna premente a disponibilidade de informações e indicadores que possibilitem identificar os problemas de saúde dos territórios. Dessa maneira, os gestores e as equipes de saúde ao desenvolverem o planejamento de ações e serviços de saúde devem empreender análise situacional, que permita o diagnóstico situacional de saúde, cujas informações podem ser sistematizadas em 'mapas de saúde', no âmbito da região de saúde5, ou em salas de situação de saúde, no âmbito local, com a finalidade de subsidiar a tomada de decisão de gestores no território ${ }^{6}$.

Ressalta-se que as primeiras experiências de estruturação de salas de situação de saúde são atribuídas ao Brasil, com base nas informações disponíveis nos SIS, como ferramenta para orientar a gestão na tomada de decisões, de modo responsável e consentâneo às necessidades de saúde da população. Reconhece-se que a inauguração da primeira sala de situação ocorreu em 1994, no município de Campina Grande, Paraíba7.

A sala de situação em saúde vem sendo utilizada como uma ferramenta que aperfeiçoa o uso da informação em saúde. Ela tem sido definida como um espaço físico ou virtual, estruturada por uma equipe de trabalho que analisa a informação sanitária para fornecer apoio à gestão em saúde, na tomada de decisões, tendo um papel fundamental durante contingências sanitárias ${ }^{4}$. Ademais, assim como outros equipamentos de saúde, a sala de situação tem um fluxo próprio que facilita a organização e realização das suas atividades. É o lugar onde a equipe de trabalho elabora a análise de situações de saúde de uma população delimitada, com base na qual estabelecem-se prioridades de saúde ${ }^{8}$.

No âmbito da Atenção Primária à Saúde (APS), a análise da situação de saúde e a definição do diagnóstico local constituem etapas do planejamento local, que deve ser realizado pela equipe que atua no território, com vistas a orientar a definição de problemas e prioridades e a implementação de ações estratégicas que 
permitam melhorar as condições de vida e saúde da população, conforme estabelecido pela Política Nacional de Atenção Básica (PNAB)9. Desse modo, a estruturação das salas de situação nas unidades básicas de saúde, construídas coletivamente, favorecem a gestão e as práticas dos profissionais, mediante a geração de conhecimento sobre a realidade sanitária local.

Não obstante, apontam-se aspectos que podem dificultar a elaboração de análises de situação de saúde densas e confiáveis, com destaque para a multiplicidade dos SIS, a não integração dos diferentes sistemas de informação, o atraso no registro de informações, a qualidade dos dados, a subnotificação de doenças e agravos, além da qualificação dos profissionais ${ }^{3-4}$. Desse modo, questiona-se: quais as contribuições dos SIS na construção da sala de situação em saúde como ferramenta de apoio à gestão local na APS?

Reconhece-se que as publicações relacionadas às contribuições dos SIS para a estruturação de salas de situação, particularmente no âmbito da APS, ainda são escassas. Assim, este estudo mostra-se relevante, uma vez que fornece embasamento teórico e prático para gestores e profissionais de saúde que atuam na APS. O objetivo deste estudo foi analisar a utilização dos Sistemas de Informação em Saúde (SIS) para a construção da sala de situação na Atenção Primária à Saúde (APS).

\section{MÉTODO}

Trata-se de uma revisão integrativa, caracterizada como método de investigação, que permite a reunião, análise e síntese de pesquisas disponíveis sobre determinados temas de forma sistematizada e rigorosa. Essa modalidade de investigação possibilita agregar distintos métodos de pesquisa e desenvolver uma visão mais ampla do tema de estudo, ao mesmo tempo em que exige uma observância acurada das análises e sínteses elabordas ${ }^{10}$.

A revisão seguiu seis etapas, a saber: 1) identificação do tema e seleção da questão de pesquisa; 2) estabelecimento dos critérios de inclusão e exclusão dos estudos; 3) seleção dos estudos; 4) categorização dos estudos selecionados; 5) análise e interpretação dos resultados; e 6) apresentação da revisão/síntese do conhecimento ${ }^{11}$.

Realizou-se a coleta de dados em dezembro de 2018, por meio de busca pareada, nas seguintes bases de dados: a) Base de Dados em Enfermagem (BDENF), acessada pelo portal da Biblioteca Virtual em Saúde; b) Literatura Latino-Americana e do Caribe em Ciências da Saúde. (Lilacs), acessada pelo portal da Biblioteca Virtual em Saúde; c) Medical Literature Analysis and Retrieval System Online (Medline), acessada pelo portal da Biblioteca Virtual em Saúde; d) Scientific Electronic Library Online (SciELO), acessada diretamente pelo portal da SciELO; e) PubMed - serviço da National Library of Medicine (NIH), acessada diretamente pelo portal da PubMed; e f) Cochrane, acessada pelo portal Cochrane.

Nas bases de dados BDENF, Lilacs, Medline e SciELO usaram-se os Descritores em Ciências da Saúde (DeCS). Cruzaram-se os seguintes descritores controlados: 'Sistemas de Informação em Saúde' OR 'Diagnóstico da Situação de Saúde’ AND ‘Atenção Primária à Saúde’. No âmbito das bases de dados PubMed e Cochrane, assumiram-se os descritores controlados pelo Medical Subject Headings (MeSH) da National Library of Medicine (NIH), adotando-se os seguintes cruzamentos: 'Health Information Systems' OR ‘Diagnosis of Health Situation’ AND ‘Primary Health Care’.

Os critérios de inclusão estabelecidos para os estudos encontrados foram: artigos disponíveis na íntegra, publicados no período de 2008-2018, nos idiomas português, inglês ou espanhol, e que respondessem à questão da pesquisa. Os critérios de exclusão foram: artigos de revisão, manuais e documentos técnicos, teses e dissertações, assim como os artigos repetidos nas bases de dados.

No processo de busca pareada, dois pesquisadores, separadamente, realizaram leitura do título e do resumo dos artigos encontrados nas bases de dados selecionadas. Caso, após essa primeira etapa, não ficasse clara ainda a pertinência do estudo, realizava-se uma leitura flutuante. Desse modo, no primeiro 
momento, foram incluídos estudos que, na avaliação dos pesquisadores, respondiam à questão norteadora. Em casos de divergência, o estudo foi avaliado por um terceiro pesquisador, que decidiu sobre a inclusão ou não do material. A síntese do processo de seleção pode ser observada no fluxograma abaixo (Figura 1).

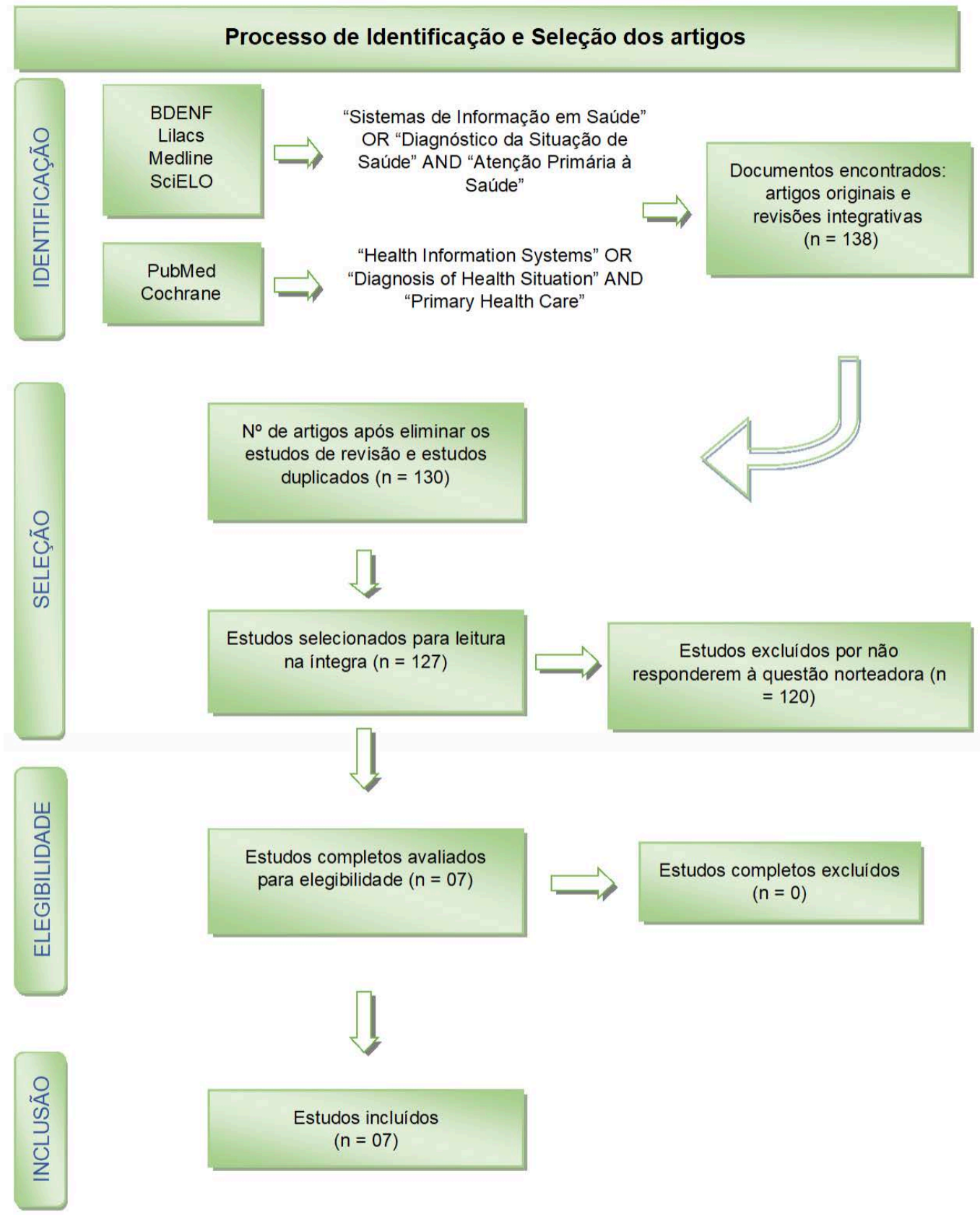

Figura 1 - Fluxograma/prisma da identificação e seleção dos artigos para o estudo Fonte: Elaborado pelos autores (2019).

Um formulário de coleta de dados foi desenvolvido e preenchido para cada artigo da amostra final do estudo. $O$ formulário permitiu a aquisição de informações para a identificação detalhada do artigo: autores e localização, objetivos, delineamento e tipo do estudo, coerência teórico-metodológica, análise dos dados, resultados e discussão, conclusões e recomendações para a prática de enfermagem.

No que concerne ao nível de evidência, os estudos foram classificados segundo o seguinte delineamento: nível 1 - evidências resultantes da metanálise de múltiplos estudos clínicos controlados e randomizados; nível 2 - evidências obtidas em estudos individuais com delineamento experimental; nível 3 - evidências de estudos quase experimentais; nível 4 - evidências de estudos descritivos (não experimentais) ou com 
abordagem qualitativa; nível 5 - evidências provenientes de relatos de caso ou de experiência; e nível 6 evidências baseadas em opiniões de especialistas ${ }^{12}$.

Com base na análise de conteúdo e temática ${ }^{13}$, organizaram-se os resultados encontrados nas seguintes categorias: a) contribuição dos SIS na coleta, processamento e divulgação de indicadores de saúde; b) o apoio dos SIS na análise e comparação de indicadores; e c) os SIS no apoio ao planejamento, à gestão e à avaliação em saúde.

\section{RESULTADOS}

Conforme demonstrado na Tabela 1, identificaram-se 138 artigos dos quais, após leitura de títulos e resumos, excluíram-se 11 (7,9\%), pois não atendiam aos critérios de inclusão. Dos 127 (92\%) artigos préselecionados na amostra parcial, após a leitura na íntegra, eliminaram-se $120(84,8 \%)$ que não respondiam à questão norteadora. Portanto, no total, excluíram-se 131 (95,6\%) artigos e a amostra final foi composta por 07 (5\%) publicações.

Tabela 1 - Distribuição dos artigos encontrados e selecionados

\begin{tabular}{cccccc}
\hline $\begin{array}{c}\text { Bases } \\
\text { bibliográficas }\end{array}$ & $\begin{array}{c}\text { Artigos } \\
\text { encontrados }\end{array}$ & $\begin{array}{c}\text { Artigos } \\
\text { excluídos }\end{array}$ & $\begin{array}{c}\text { Amostra } \\
\text { parcial }\end{array}$ & $\begin{array}{c}\text { Excluídos da } \\
\text { amostra parcial }\end{array}$ & $\begin{array}{c}\text { Amostra } \\
\text { final }\end{array}$ \\
\hline BDENF & 4 & 2 & 2 & 0 & 2 \\
Lilacs & 56 & 2 & 54 & 53 & 1 \\
Medline & 20 & 3 & 17 & 16 & 1 \\
SciELO & 15 & 1 & 14 & 12 & 2 \\
Cochrane & 1 & 0 & 1 & 1 & 0 \\
PubMed & 42 & 3 & 39 & 38 & 1 \\
TOTAL & $\mathbf{1 3 8}$ & $\mathbf{1 1}$ & $\mathbf{1 2 7}$ & $\mathbf{1 2 0}$ & $\mathbf{7}$ \\
\hline
\end{tabular}

Fonte: Elaborado pelos autores (2019).

A leitura e análise dos artigos selecionados permitiram uma síntese de acordo com autor, título, periódico e país de publicação, objetivo e método, bem como o nível de evidência do estudo, conforme apresentado no Quadro 1. 
Quadro 1 - Síntese dos artigos publicados nas bases de dados na última década

\begin{tabular}{|c|c|c|c|c|}
\hline $\begin{array}{c}\text { Base de } \\
\text { dados/ } \\
\text { Biblioteca } \\
\text { virtual (ano) }\end{array}$ & Título & Periódico/país & Objetivo & $\begin{array}{l}\text { Método (Nível } \\
\text { de evidência) }\end{array}$ \\
\hline $\begin{array}{l}\text { (A1) } \\
\text { BDENF (2016) }\end{array}$ & $\begin{array}{l}\text { Informações em } \\
\text { saúde na ótica de } \\
\text { enfermeiras da } \\
\text { Saúde da Família }\end{array}$ & $\begin{array}{l}\text { Revista Mineira } \\
\text { de Enfermagem/ } \\
\text { Brasil }\end{array}$ & $\begin{array}{l}\text { Analisar a utilidade e a utilização } \\
\text { das informações em saúde como } \\
\text { ferramenta para organizar o } \\
\text { processo de trabalho, a partir } \\
\text { da ótica de enfermeiras que } \\
\text { trabalham na Saúde da Família, } \\
\text { especificamente nos municípios } \\
\text { de Campinas-SP e Dourados-MS. }\end{array}$ & $\begin{array}{l}\text { Estudo descritivo/ } \\
\text { qualitativo } \\
\text { (Nível de } \\
\text { evidência 4) }\end{array}$ \\
\hline $\begin{array}{l}(\text { A2) } \\
\text { BDENF (2011) }\end{array}$ & $\begin{array}{l}\text { Fluxo informacional } \\
\text { do Sistema de } \\
\text { Informação da } \\
\text { Atenção Básica: } \\
\text { vigilância e } \\
\text { centralização }\end{array}$ & $\begin{array}{l}\text { Revista de } \\
\text { Enfermagem do } \\
\text { Centro-Oeste } \\
\text { Mineiro/Brasil }\end{array}$ & $\begin{array}{l}\text { Analisar o fluxo informacional } \\
\text { do SIAB, a partir de unidades da } \\
\text { Saúde da Família até os níveis } \\
\text { centrais (Secretarias Municipal e } \\
\text { Estadual de Saúde; Ministério da } \\
\text { Saúde). }\end{array}$ & $\begin{array}{l}\text { Estudo descritivo- } \\
\text { exploratório/ } \\
\text { qualitativo } \\
\text { (Nível de } \\
\text { evidência 4) }\end{array}$ \\
\hline $\begin{array}{l}\text { (A3) } \\
\text { Medline (2016) }\end{array}$ & $\begin{array}{l}\text { Sistemas de } \\
\text { Informação } \\
\text { em Saúde: } \\
\text { inconsistências } \\
\text { de informações } \\
\text { no contexto da } \\
\text { atenção primária }\end{array}$ & $\begin{array}{l}\text { Journal of Health } \\
\text { Informatics/Brasil }\end{array}$ & $\begin{array}{l}\text { Comparar os dados referentes } \\
\text { a hipertensão, diabetes, } \\
\text { tuberculose e hanseníase entre o } \\
\text { SIAB e os sistemas SisHiperdia e } \\
\text { SINAN. }\end{array}$ & $\begin{array}{l}\text { Estudo } \\
\text { transversal, } \\
\text { descritivo/ } \\
\text { quantitativo } \\
\text { (Nível de } \\
\text { evidência 4) }\end{array}$ \\
\hline $\begin{array}{l}\text { (A4) } \\
\text { SciELO (2015) }\end{array}$ & $\begin{array}{l}\text { Sistema de } \\
\text { Informação da } \\
\text { Atenção Básica: } \\
\text { potencialidades } \\
\text { para a promoção } \\
\text { da saúde }\end{array}$ & $\begin{array}{l}\text { Acta Paulista de } \\
\text { Enfermagem/ } \\
\text { Brasil }\end{array}$ & $\begin{array}{l}\text { Discutir a utilização do Sistema } \\
\text { de Informação da Atenção Básica } \\
\text { na perspectiva da promoção da } \\
\text { saúde. }\end{array}$ & $\begin{array}{l}\text { Estudo descritivo/ } \\
\text { quantitativo } \\
\text { (Nível de } \\
\text { evidência 4) }\end{array}$ \\
\hline $\begin{array}{l}\text { (A5) } \\
\text { PubMed (2013) }\end{array}$ & $\begin{array}{l}\text { Decline in } \\
\text { mortality with the } \\
\text { Belize Integrated } \\
\text { Patient-Centred } \\
\text { Country Wide } \\
\text { Health Information } \\
\text { System (BHIS) } \\
\text { with embedded } \\
\text { program } \\
\text { management }\end{array}$ & $\begin{array}{l}\text { International } \\
\text { Journal of } \\
\text { Medical } \\
\text { Informatics/ } \\
\text { Canadá }\end{array}$ & $\begin{array}{l}\text { Avaliar a adesão do BHIS } \\
\text { por profissionais de saúde, } \\
\text { a mortalidade antes e após } \\
\text { a implantação do BHIS em } \\
\text { determinadas áreas e sua relação } \\
\text { com as despesas de saúde } \\
\text { pública. }\end{array}$ & $\begin{array}{l}\text { Estudo descritivo } \\
\text { (Nível de } \\
\text { evidência 4) }\end{array}$ \\
\hline $\begin{array}{l}\text { (A6) } \\
\text { Lilacs (2014) }\end{array}$ & $\begin{array}{l}\text { A sala de situação } \\
\text { da dengue como } \\
\text { ferramenta de } \\
\text { gestão em saúde }\end{array}$ & $\begin{array}{l}\text { Saúde em } \\
\text { Debate/Brasil }\end{array}$ & $\begin{array}{l}\text { Apresentar a sala de situação } \\
\text { da dengue como ferramenta de } \\
\text { apoio ao planejamento e à gestão } \\
\text { de saúde no Distrito Sanitário IV, } \\
\text { em João Pessoa, Paraíba. }\end{array}$ & $\begin{array}{l}\text { Estudo } \\
\text { documental } \\
\text { (Nível de } \\
\text { evidência 4) }\end{array}$ \\
\hline $\begin{array}{l}\text { (A7) } \\
\text { SciELO (2016) }\end{array}$ & $\begin{array}{l}\text { Painel de } \\
\text { monitoramento } \\
\text { municipal: bases } \\
\text { para a construção } \\
\text { de um instrumento } \\
\text { de gestão dos } \\
\text { serviços de saúde }\end{array}$ & $\begin{array}{l}\text { Epidemiologia } \\
\text { e Serviços de } \\
\text { Saúde/Brasil }\end{array}$ & $\begin{array}{l}\text { Descrever e analisar criticamente } \\
\text { os caminhos percorridos e o } \\
\text { referencial técnico utilizado } \\
\text { na construção do Painel de } \\
\text { monitoramento da Secretaria } \\
\text { Municipal da Saúde de São } \\
\text { Paulo (SMS-SP), destacar suas } \\
\text { potencialidades e limites para a } \\
\text { tomada de decisão. }\end{array}$ & $\begin{array}{l}\text { Estudo qualitativo } \\
\text { (Nível de } \\
\text { evidência 4) }\end{array}$ \\
\hline
\end{tabular}

Fonte: Elaborado pelos autores (2019). 
Ao analisar os artigos, observou-se que 71,4\% dos estudos selecionados foram publicados entre os anos de 2011 a 2016, em periódicos distintos, cujos países de origem são o Brasil (85,7\%) e o Canadá (14,3\%). No que tange à abordagem metodológica dos trabalhos, percebeu-se que todos os estudos eram descritivos ou qualitativos referindo-se ao nível de evidência 4 (Quadro 1).

Na sequência, apresenta-se a relação das categorias formuladas com base na similaridade de conteúdo dos artigos (Quadro 2).

\section{Quadro 2 - Organização dos artigos em categorias, segundo similaridade de conteúdo}

\begin{tabular}{|l|l|}
\hline \multicolumn{1}{|c|}{ Categoria } & \multicolumn{1}{c|}{ Artigos } \\
\hline $\begin{array}{l}\text { 1. Contribuição dos Sistemas de Informação em Saúde na } \\
\text { coleta, processamento e divulgação de indicadores de saúde }\end{array}$ & A1; A4; A6 \\
\hline $\begin{array}{l}\text { 2. Apoio dos Sistemas de Informação em Saúde na análise e } \\
\text { comparação de indicadores }\end{array}$ & A2; A4; A6; A7 \\
\hline $\begin{array}{l}\text { 3. Os Sistemas de Informação em Saúde no apoio ao } \\
\text { planejamento, gestão e avaliação em saúde }\end{array}$ & A1; A3; A4; A5; A6; A7 \\
\hline
\end{tabular}

Fonte: Elaborado pelos autores (2019).

Elencaram-se os resultados encontrados em três categorias, conforme mostra o Quadro 2. Após a análise exaustiva dos dados de cada estudo, três artigos $(42,8 \%)$ enquadraram-se na primeira categoria, quatro $(57,1 \%)$ na segunda e seis $(85,7 \%)$ na terceira categoria. Vê-se ainda que alguns estudos foram alocados em mais de uma categoria.

\section{DISCUSSÃO}

\section{Contribuição dos Sistemas de Informação em Saúde na coleta, no processamento e na divulgação de indicadores de saúde}

No que tange à primeira categoria, que foca na contribuição dos SIS na coleta, tabulação e no processamento de dados para produzir planilhas de indicadores e gráficos dentro da sala de situação, enfatizaram-se os seguintes pontos que os autores relacionam aos sistemas:

- tornam as informações, antes armazenadas, disponíveis eletronicamente ${ }^{14}$;

- geram tabelas de produção ${ }^{15-16}$.

Acredita-se que essas funções dos SIS são utilizadas dentro da sala de situação, uma vez que as informações armazenadas nos sistemas eletrônicos e os relatórios de produção gerados na análise têm o potencial de instrumentalizar os processos de gestão e atenção à saúde, mediante a construção, análise e divulgação dos indicadores de saúde.

Uma das principais finalidades da sala de situação em saúde é coletar, tabular e realizar o processamento de dados para produção de planilhas de indicadores e gráficos ${ }^{14}$. Logo, os SIS são relevantes nesse contexto, uma vez que os dados e as tabelas de produção, disponibilizados eletronicamente pelos próprios sistemas, permitem que os profissionais inseridos na sala de situação em saúde possam realizar levantamentos e comparações, bem como analisar gráficos, o que contribui diretamente na produção de indicadores de saúde ${ }^{15}$.

A produção, a divulgação da informação e o seu gerenciamento são subsídios estratégicos voltados tanto para o fortalecimento de objetivos e ações das organizações quanto para a eficácia das ações de controle social com base em indicadores e gráficos de saúde. Contudo, percebe-se que um dos grandes desafios da 
gestão efetiva da APS, além da sua estruturação, é também a disponibilização de informações confiáveis, adequadas e personalizadas ${ }^{17}$. Então, a produção de planilhas de indicadores e de gráficos na sala de situação é fundamental para apoiar as decisões da administração pública e o planejamento estratégico alinhado às necessidades da população.

A divulgação dos indicadores de saúde dentro dos SIS torna cada vez mais possível a percepção do quadro real de saúde de uma população, configurando-se como um meio eficaz para a tomada de decisão em saúde tanto por parte dos profissionais, no âmbito da APS, como também pelos gestores dos serviços de saúde ${ }^{18}$.

Além disso, as tabelas e os gráficos formulados pelos SIS consistem em importantes ferramentas para avaliar o uso adequado dos recursos oriundos do SUS, tal como a qualidade da assistência prestada à população em geral. Dessa forma, os SIS tornam-se fundamentais para os processos de auditoria realizados no âmbito do SUS ${ }^{19}$.

Por conseguinte, estabelece-se entre os SIS e a sala de situação em saúde uma relação que deve ser fomentada, discutida e estudada como uma ferramenta estratégica de gestão, principalmente em nível de APS. Com efeito, os relatórios gerados e a disponibilização das informações nos sistemas de informação apoiam diretamente a construção e a análise de indicadores de saúde na sala de situação.

\section{Apoio dos Sistemas de Informação em Saúde na análise e comparação de indicadores}

Em relação às atividades dos SIS que podem contribuir na análise e comparação de dados na sala de situação, destacaram-se os seguintes pontos:

- geram relatórios de análise de dados ${ }^{15}$

- possibilitam a análise dessas informações de situação de saúde, no nível local, seja no espaço físico ou virtual ${ }^{16,20-21}$.

Nessa categoria, os autores defendem a ideia de que os relatórios de análises de dados, gerados pelas informações já coletadas e tabuladas, contribuem diretamente para novas análises e para comparações das informações em tempo real e rápido, quer seja dentro de um espaço bem estruturado ou no próprio sistema virtual.

A sala de situação em saúde, como espaço onde se articulam análises e comparações de $\operatorname{dados}^{6}$, recebe suporte dos SIS, visto que os dados são disponibilizados pelos sistemas - o que favorece, de forma rápida e segura, a transformação de um dado bruto em uma informação relevante. Cabendo, claro, aos profissionais da sala, dentre eles o enfermeiro da unidade básica de saúde, analisarem de forma crítica, horizontal e cuidadosa tais informações a fim de nortear ações de saúde.

Na ambiência da APS, esses relatórios de análise de dados na sala de situação possibilitam o entendimento das condições que influenciam no processo saúde-doença da comunidade. Dessa maneira, permitem a identificação e compreensão das possíveis mudanças de riscos e os agravos que acometem essa população²2.

No que concerne ao nível local, esses relatórios e indicadores apoiam o planejamento e a organização de ações em saúde específicas para a comunidade. É somente a partir do conhecimento da situação de saúde vivenciada que se torna possível selecionar os problemas de saúde prioritários e assim traçar estratégias de intervenções coerentes para a melhoria das condições de saúde da população ${ }^{23}$.

Alguns autores descrevem que há ainda no Brasil muitas unidades de saúde com um sistema precário de informações, o que inibe a interoperabilidade e faz com que diversos cadastros sejam preenchidos equivocadamente com informações semelhantes. Sem falar também no déficit de recursos humanos, como, por exemplo, a carência de auxiliares administrativos para a digitação correta dos dados e para a alimentação do sistema ${ }^{24}$. Essa dificuldade remete à necessidade de se discutir, entre os sujeitos que 
compõem a equipe multiprofissional na APS, sobre a importância da atualização e veracidade dos dados a serem disponibilizados, enfatizando a conscientização e o compromisso no preenchimento adequado e completo das fichas que estruturam o trabalho das equipes de APS.

Por isso, apesar dos grandes avanços dos SIS, ao longo dos anos, a realização de análises e comparações, nas salas de situação, ainda é um desafio, considerando-se, sobretudo, as falhas existentes com relação à confiabilidade dos dados.

\section{Os Sistemas de Informação em Saúde no apoio ao planejamento, à gestão e à avaliação em saúde}

Apreendeu-se que as ações desenvolvidas na operacionalização dos SIS contribuem para a avaliação de problemas, para as intervenções e a tomada de decisões, mediante o suporte da sala de situação. Desse modo, os SIS representam uma ferramenta que possibilita qualificar os processos gerenciais e assistenciais na APS, uma vez que sua operacionalização, no contexto da sala de situação, subsidia a atuação das equipes nas seguintes dimensões:

- na mudança do modelo assistencial ${ }^{14}$;

- no reconhecimento do perfil socioeconômico e sanitário da população/território ${ }^{15}$;

- no processo de organização, análise, transmissão e divulgação de dados de saúde ${ }^{14-15}$;

- na definição de áreas prioritárias para intervenção $0^{14,16}$;

- na construção de indicadores de saúde ${ }^{21}$;

- na gestão do trabalho dos profissionais, pois auxilia a organização das equipes 25-26. $^{2}$.

Com base no exposto, infere-se que os SIS são ferramentas norteadoras ${ }^{16}$ da gestão e do trabalho em saúde. A utilização dos SIS nas salas de situação instrumentaliza as práticas avaliativas, com foco na tomada de decisões. Denota-se, ainda, contribuições dos SIS na organização das equipes de planejamento, de vigilância epidemiológica, de informática em saúde, além das equipes de atenção à saúde no território da APS. Assim, a operacionalidade das salas de situação, no âmbito da APS, permite a qualificação das práticas de saúde, ampliando a efetividade do serviço de saúde, sobretudo na prevenção e no controle de riscos e agravos à saúde dos indivíduos ${ }^{6}$.

Ressalta-se, ainda, que os SIS viabilizam planejamento, gestão, avaliação e controle nos serviços e ações de saúde ${ }^{27}$. Por isso, conferem direcionalidade à gestão e às equipes de saúde no desenvolvimento de ações de alcance individual e coletivo, considerando-se as áreas programáticas e os grupos populacionais prioritários, definidos com base nos critérios de riscos e vulnerabilidades. Logo, compete à equipe e, particularmente, ao enfermeiro que organiza a sala de situação, empreender análises de situação de saúde e tomar decisões que produzam impacto positivo no contexto que necessita de mudanças.

A informação é essencial para a gestão e para o controle social do SUS, cujas análises estatísticas sanitárias, epidemiológicas e de atividades de produção de serviço, abrangendo toda a complexidade dos serviços de saúde, têm sido asseguradas por meio da gerência informatizada ${ }^{28}$. Assim, os SIS têm auxiliado os gestores e profissionais da sala de situação em saúde na identificação de problemas, o que permite avaliações eficazes no direcionamento da tomada de decisão e na instrumentalização das esferas de controle social ${ }^{29}$. O enfermeiro, como gestor inserido na sala, tem contribuído diretamente nesse processo, pois ele integra a equipe e com ela compartilha um rol de ações comuns, incluindo a análise de situação de saúde e a proposição de intervenções diretas na APS.

A estruturação da sala de situação em saúde impõe a criação de grupos de trabalho com profissionais de planejamento, informática, da área epidemiológica, pois a operacionalização dos SIS, por conta da 
complexidade, requer o conhecimento de diversas áreas. Em consequência, observa-se uma demanda por qualificação para o gerenciamento das atividades das salas de situação. Destaca-se, sobretudo, a importância da proposição, por parte do gestor, de fluxos de informação adequados, no que se refere à integração necessária entre vigilância e atenção à saúde.

No âmbito da APS, enfatiza-se que os SIS, especialmente o Sistema de Informação em Saúde para Atenção Básica (SISAB), atualmente substituído pelo e-SUS Atenção Básica, têm sido bastante utilizados como fonte de informações sobre cadastros de famílias, condições de moradia, saneamento, situação de saúde, produção e composição das equipes de saúde, tornando-se necessário para que municípios de pequeno porte e com precariedade de dados possam fazer uma cobertura de atenção primária adequada.

Os estudos não afirmaram explicitamente que o SISAB tenha sido um dos principais sistemas utilizados para a estruturação das salas de situação na APS, porém acredita-se que, na prática, este seja o sistema de informação mais operacionalizado pelas equipes, uma vez que é específico dessa área e abrange um conjunto de informações relacionadas ao perfil populacional do território e ao processo de trabalho das equipes, que são responsáveis pela coleta de dados e por inseri-los, regularmente, no sistema.

\section{CONCLUSÃO}

Os artigos incluídos neste estudo e as evidências e discussões aqui apresentadas apontam para as contribuições dos SIS na organização das salas de situação em saúde, que constituem uma ferramenta de apoio à gestão local. Nesse sentido, as salas permitem os diagnósticos de saúde no território, na construção de indicadores, no planejamento e na avaliação, bem como na organização dos processos de trabalho, considerando a definição das ações desenvolvidas pelas equipes de saúde, tornando eficazes as tomadas de decisões dos gestores na APS.

Diante disso, reconhece-se a importância dos SIS e das salas de situação, sendo a articulação entre ambos necessária para qualificar os processos de gestão na APS. Cabe à equipe inserida nesse contexto apropriarse dessa ferramenta para alcançar competências gerenciais. Sendo assim, a equipe poderá administrar a complexidade existente dentro do ambiente, identificando e buscando aprimorar os processos de gestão e atenção à saúde, mediante a incorporação da sala de situação como ferramenta gerencial.

Os resultados desta pesquisa contribuem significativamente para que gestores e profissionais de saúde compreendam a importância da sala de situação, já que expusemos aqui de que forma os SIS têm contribuído para as atividades realizadas nessas salas, sejam elas físicas ou virtuais, deixando espaço ainda para que esses profissionais reflitam sobre possíveis melhorias necessárias nesse contexto.

Os limites deste estudo estão relacionados à escassez de material sobre a temática, evidenciada nas bases de dados. Isso, por sua vez, aponta a necessidade de mais pesquisas sobre como os profissionais e gestores da APS incorporam as contribuições da sala de situação nos seus processos de trabalho, considerando a reorientação do modelo de atenção à saúde. Recomenda-se, ainda, o desenvolvimento de estudos em que se avalie a gestão da informação em saúde, considerando-se a problemática da fragmentação e da confiabilidade dos dados sinalizada neste artigo.

\section{REFERÊNCIAS}

1. Pinochet LHC, Lopes AS, Silva JS. Inovações e tendências aplicadas nas tecnologias de informação e comunicação na gestão da saúde. Rev Gest Sist Saúde. 2014;3(2):11-29.

2. Araújo LO, Razzolini FE. Os sistemas de informação como suporte à tomada de decisão estratégica. Rev Compet Sustentabilidade. 2017;4(2):66-75. 
3. $\quad$ Pinto LF, Freitas MPS, Figueiredo AWS. Sistemas Nacionais de Informação e levantamentos populacionais: algumas contribuições do Ministério da Saúde e do IBGE para a análise das capitais brasileiras nos últimos 30 anos. Ciênc Saúde Colet. 2018;23(6):1859-70.

4. Conselho Nacional de Secretários de Saúde (BR). Sistema Único de Saúde. Brasília: CONASS; 2007.

5. Decreto no 7.508 (BR), de 28 de junho de 2011. Regulamenta a Lei no 8.080, de 19 de setembro de 1990, para dispor sobre a organização do Sistema Único de Saúde - SUS, o planejamento da saúde, a assistência à saúde e a articulação interfederativa, e dá outras providências. DOU [Internet]. 2011 [citado em 2020 jul. 30]. Disponível em: http://www.plana Ito.gov.br/ccivil 03/Ato2011-2014/2011/ Decreto/D7508.htm.

6. Lucena KDT, Deininger LS, Silva EA, Figueiredo DCM, Pereira AJ, Vianna RPT. Health situation room as a tool for management: planning the territory of shares. Rev Enferm UFPE on line. 2014;8(3):702-8.

7. Moya J. Panorama sobre as salas de situação de saúde na América Latina e Caribe. In: Moya J, Risi Junior JB, Martinello A, Bandarra E, Bueno H, Morais Neto OL, organizadores. Sala de situação em saúde: compartilhando as experiências do Brasil. Brasília: Organização Pan-Americana da Saúde; 2010. p. $49-60$.

8. Sousa FAMR, Goulart MJG, Braga AMS, Medeiros CMO, Rego DCM, Vieira FG et al. Setting health priorities in a community: a case example. Rev Saúde Pública. 2017;51:1-10.

9. Ministério da Saúde (BR). Portaria no 2.436, de 21 de setembro de 2017. Aprova a Política Nacional de Atenção Básica, estabelecendo a revisão de diretrizes para a organização da Atenção Básica, no âmbito do Sistema Único de Saúde (SUS). DOU [Internet]. 2017 [citado em 2020 jul. 28]. Disponível em: https://bvsms.saude.gov.br/bvs/saudelegis/gm/2017/prt2436 2209 2017.html.

10. Lockwood C, Porrit K, Munn Z, Rittenmeyer L, Salmond S, Bjerrum M et al. Revisões sistemáticas de evidências qualitativas. In: Aromataris E, Munn Z, editores. Manual da JBI para síntese de evidências. JBI; 2020.

11. Mendes KDS, Silveira RCCP, Galvão CM. Revisão integrativa: método de pesquisa para a incorporação das evidências na saúde e na enfermagem. Texto Contexto Enferm. 2008;17(4):758-764.

12. Pedrosa KKA, Oliveira ICM, Feijão AR, Machado RC. Enfermagem baseada em evidência: caracterização dos estudos no Brasil. Cogitare Enferm. 2015;20(4):733-741.

13. Brechós $\mathrm{B}$, Ribeiro VM, Vóvio $\mathrm{CL}$, Haas $\mathrm{CM}$. Procedimentos metodológicos para se eleger categorias de análise baseadas em referências teórico-conceituais. Educ Fronteiras. 2018;8(22):221-35.

14. Martins LMP, Silva EM, Marques D. Health information in the perspective of family health nurses. Rev Min Enferm. 2016;20:e932.

15. Schülter BHIT, Baeta NACMF, Vieira HPM, Cossentino BMC, Costa CC. Primary care information system: potential for health promotion. Acta Paul Enferm. 2015;28(2):152:9.

16. Deininger LDSC, Lucena KDTD, Figueiredo DCMMD, Silva CCD, Oliveira AECD, Anjos UUD. A sala de situação da dengue como ferramenta de gestão em saúde. Saúde Debate. 2014;38(100):50-6.

17. Pinto LF, Rocha CMF. Innovations in Primary Health Care: the use of communications technology and information tools to support local management. Ciênc. Saúde Colet. 2016;21(5):1433-48.

18. Lima KWS, Antunes JLF, Silva ZP. Percepção dos gestores sobre o uso de indicadores nos serviços de saúde. Saúde Soc. 2015;24(1):61-71.

19. Brandão ACS, Silva JRA. A contribuição dos Sistemas de Informação em Saúde (SIS) para o processo de auditoria do SUS. Rev Eletrôn Atualiza Saúde. 2015;1(1):17-24.

20. Cavalcante RB, Pinheiro MMK, Bernardes MFVG, Cunha SGS, Santos CS. Fluxo informacional do Sistema de Informação da Atenção Básica: vigilância e centralização. Rev Enferm Cent O Min. 2011;1(4):523-36.

21. Grimm SCDA, Tanaka OY. Painel de monitoramento municipal: bases para a construção de um instrumento de gestão dos serviços de saúde. Epidemiol Serv Saúde. 2016;25(3):585-94.

22. Feitosa RMM, Almeida ANS, Silva WF, Xavier MSM. Situation room in health: planning tool for health actions. Rev Enferm UFPE on line. 2014;8(7):2165-70.

23. Cordeiro TMSC, Neto JNC. Situação de saúde de um município do interior da Bahia: uma proposta de intervenção. Rev Bras Pesq Saúde. 2017;19(4):23-33. 
24. Correia LOS, Padilha BM, Vasconcelos SML. Métodos para avaliar a completitude dos dados dos Sistemas de Informação em Saúde do Brasil: uma revisão sistemática. Ciênc Saúde Colet. 2014;19(11):4467-78.

25. Pereira BS, Tomasi E. Instrumento de apoio à gestão regional de saúde para monitoramento de indicadores de saúde. Epidemiol Serv Saúde. 2016;25(2):411-18.

26. Araújo YB, Rezende LCM, Queiroga MMD, Santos SR. Sistemas de Informação em Saúde: inconsistências de informações no contexto da Atenção Primária. J Health Inform. 2016;8(5):164-70.

27. Neves TCCL, Montenegro LA, Bittencourt SDA. Produção e registro de informações em saúde no Brasil: panorama descritivo através do PMAQ-AB. Saúde Debate. 2014;103(38):756-70.

28. Lima AC, Januário MC, Lima PT, Silva WM. DATASUS: o uso dos sistemas de informação na saúde pública. Refas. 2015;1(3):16-31.

29. Pinheiro ALS, Andrade KTS, Silva DO, Zacharias FCM, Gomide MFS et al. Health management: the use of information systems and knowledge sharing for the decision making process. Texto Context Enferm. 2016;25(3):e3440015. 